\title{
QUASI-INVARIANCE OF ANALYTIC MEASURES ON COMPACT GROUPS ${ }^{1,2}$
}

\author{
BY V. MANDREKAR ${ }^{3}$ AND M. NADKARNI \\ Communicated by H. Helson, May 26, 1967
}

1. Introduction. The study of analytic measures on compact groups with ordered duals has been the subject of several papers on Fourier Analysis in recent years (see W. Rudin [12] for references). In their papers [5], [6], H. Helson and D. Lowdenslager have used a new method to study the properties of analytic functions on the Bohr group. In his subsequent works [3], [4], Helson has emphasized the connection of this problem to the Weyl-Von Neumann operator equations $([8],[10])$. In the meantime, K. de Leeuw and I. Glicksberg [2] have given an extension of the classical theorem of $F$. and M. Riesz to compact groups. They obtain as its consequence refinements of some theorems of Helson-Lowdenslager [5] and S. Bochner [1].

Our purpose here is to use Helson's method in [4] to obtain a simple proof of the de Leeuw-Glicksberg theorem basing ourselves entirely on the Hilbert space geometry. We think that the interest of this proof, aside from its simplicity and clarity, lies in unifying the ideas of the above two approaches. This unity may eventually lead to a deeper knowledge of analytic measures on groups with ordered duals. Such a study has been made in the special case of the Bohr group by M. G. Nadkarni [9]. A complete study may also give an extension of the work of G. Kallianpur and V. Mandrekar [7] to the situation considered by Helson-Lowdenslager [6].

Professors K. de Leeuw and I. Glicksberg have brought to our notice the yet unpublished work, Analytic and quasi-invariant measures by Frank Forelli, where he defines analytic measures on an arbitrary locally compact Hausdorff space and studies their quasiinvariance. His work has points of contact with our work; however, it being more general, he needs elaborate techniques in the theory of Abelian group algebras.

${ }^{1}$ Prepared with the support of a grant from the United States Air Force Office of Scientific Research-Office of Aerospace Research, Grant No. AF-AFOSR-885-65.

2 This paper appeared recently in Acta Mathematica 118 (1967), 33-57.

${ }^{3}$ Presently at Math Research Center, Madison, Wisconsin. 
2. Quasi-invariance of spectral measures. Let $G$ be a locally compact Abelian group. Let $\Gamma$ be the character group of $G$. Let $H$ be a Hilbert space. Let $\left\{U_{\gamma}: \gamma \in \Gamma\right\}$ be a strongly continuous group of unitary operators on $H$. It is known $[11$, p. 392] that there exists a hermitian projection valued measure $\beta$ on the Borel subsets of $G$ such that

$$
U_{\gamma}=\int_{G} \chi_{\gamma}(g) \beta(d g)
$$

where $\chi_{\gamma}$ denotes the character on $G$ corresponding to $\gamma \in \Gamma$.

Let $\psi$ be a continuous homomorphism of $\Gamma$ into $R$, the group of real numbers with the usual topology. $\psi$ induces a homomorphism $\phi: R \rightarrow G$ of the associated dual groups. In fact, $\phi$ is the unique mapping defined by $\chi_{\gamma}(\phi(t))=\exp (i \psi(\gamma) t)$. With the above notation we obtain the following result of purely geometric nature which will be used in $\S 3$ to prove the de Leeuw-Glicksberg theorem.

THEOREM 2.1. Let $\left\{V_{t} ; t \in R\right\}$ be a group of unitary operators satisfying

$$
U_{\gamma} V_{t}=\chi_{\gamma}(\phi(t)) V_{t} U_{\gamma} .
$$

Then $V_{t} \beta(\Delta) V_{-t}=\beta(\Delta+\phi(t))$ where $\beta$ is the spectral measure on $G$ corresponding to $\left\{U_{\gamma}, \gamma \in \Gamma\right\}$ and $\Delta$ is any Borel subset of $G$.

Proof. By (2.1) we have

$$
U_{\gamma} V_{t}=\int_{G} \chi_{\gamma}(g) \beta(d g) V_{t}
$$

But

$$
\begin{aligned}
\chi_{\gamma}(\phi(t)) V_{t} U_{\gamma} & =\chi_{\gamma}(\phi(t)) V_{t} \int_{G} \chi_{\gamma}(g) \beta(d g)=\chi_{\gamma}(\phi(t)) \int_{G} \chi_{\gamma}(g) V_{t} \beta(d g) \\
& =\int_{G} \chi_{\gamma}\left(g+(\phi(t)) V_{t} \beta(d g)\right)=\int_{G} \chi_{\gamma}(g) V_{t} \beta(d g-\phi(t)) .
\end{aligned}
$$

Since $U_{\gamma} V_{t}=\chi_{\gamma}(\phi(t)) V_{t} U_{\gamma}$, we can equate (2.3) and (2.4) to obtain

$$
\int_{G} \chi_{\gamma}(g) \beta(d g) V_{t}=\int_{G} \chi_{\gamma}(g) V_{t} \beta(d g-\phi(t))
$$

i.e., for all $x, y \in H$,

$$
\int_{G} \chi_{\gamma}(g)\left(\beta(d g) V_{t} x, y\right)=\int_{G} \chi_{\gamma}(g)\left(V_{t} \beta(d g-\phi(t)) x, y\right) .
$$


By the uniqueness of the Fourier transform it follows that for all $x, y \in H,\left(\beta(\Delta) V_{t} x, y\right)=\left(V_{t} \beta(\Delta-\phi(t)) x, y\right)$. In other words, $\beta(\Delta) V_{t}$ $=V_{t} \beta(\Delta-\phi(t))$. Hence $V_{t} \beta(\Delta) V_{-t}=\beta(\Delta+\phi(t))$. q.e.d.

3. Quasi-invariance of analytic measures. Let $G$ be a compact Abelian group and $\Gamma$ its discrete dual. An "ordering" of $\Gamma$ is given by a fixed nontrivial homomorphism $\psi$ of $\Gamma$ into the group of real numbers. Since $\Gamma$ is discrete the mapping $\psi$ is a continuous homomorphism and thus induces a continuous homomorphism $\phi: R \rightarrow G$ of the associated dual groups; $\phi$ is the unique mapping defined by $x_{\gamma}(\phi(t))=\exp (i \psi(\gamma) t)$, $t \in R, \gamma \in \Gamma$.

Let $\mu$ be a finite complex regular measure on the Borel subsets of $G$. $\mu$ is said to be $\phi$-analytic if $\int_{G \chi_{\gamma}}(g) \mu(d g)=0$ whenever $\psi(\gamma)<0$. Let $|\mu|$ denote the total variation measure associated with $\mu$. It is easy to see that $\mu(d g)=e(g)|\mu|(d g)$ where $e(\cdot)$ is a complex valued measurable function on $G$ of absolute value 1 almost everywhere $[|\mu|] . \mu$ is called quasi-invariant under $\phi$ if $|\mu|(\Delta)=0$ implies $|\mu|(\Delta+\phi(t))=0$ for all $t \in R$. We shall denote by $L_{2}(G,|\mu|)$ the space of complexvalued functions square integrable with respect to $|\mu|$, where functions equal a.e. $[|\mu|]$ are identified. We consider the subspaces $\mathfrak{M T}_{\mathrm{s}}$ of $L_{2}(G,|\mu|)$ generated by $\left\{\chi_{\gamma}(\cdot) e(\cdot): \psi(\gamma) \leqq s\right\}$ for each $s$. They have the following properties:

$$
\begin{aligned}
& \text { (i) } \mathscr{T}_{s} \leqq \Re_{u} \text { if } s \leqq u, \\
& \text { (ii) } \bigvee_{s} \mathbb{M}_{s}=L_{2}(G,|\mu|), \\
& \text { (iii) } \bigcap_{s} \mathscr{T}_{s}=\{0\}
\end{aligned}
$$

(i) and (ii) are obvious; only property (iii) needs a proof. Consider $\int_{a} \chi_{\gamma}(g) \chi_{\tau}(g) e(g)|\mu|(d g)$. If $\psi(\tau) \leqq-t$, then for $\gamma$ satisfying $\psi(\gamma)<t$ we have $\int_{G} \chi_{\gamma}(g) \chi_{\tau}(g) e(g)|\mu|(d g)=0$ by $\phi$-analyticity of $\mu$. Since $\mathfrak{M T}_{-t}$ is spanned by $\left\{\chi_{\tau}(\cdot) e(\cdot): \psi(\tau) \leqq-t\right\}$, we have for any $f \in \mathbb{N}_{-t}$, $\int_{G} \chi_{\gamma}(g) f(g) e(g)|\mu|(d g)=0$ for $\gamma$ with $\psi(\gamma)<t$. Let $f \in \mathbb{M}_{-\infty}$. Then $f \in \mathscr{M}_{-t}$ for each $t$. Hence we get $\int_{a} \chi_{\gamma}(g) f(g) e(g)|\mu|(d g)=0$ for all $\gamma$. This implies $f=0$ a.e. $[|\mu|]$ proving (iii).

Let $U_{\gamma}$ be the operator in $L_{2}(G,|\mu|)$ such that $U_{\gamma} f=\chi_{\gamma} f$. Then for each $\gamma \in \Gamma, U_{\gamma}$ is obviously unitary and $\left\{U_{\gamma}: \gamma \in \Gamma\right\}$ is a group of unitary operators on $L_{2}(G,|\mu|)$. Let $E(s)$ denote the orthogonal projection from $L_{2}(G,|\mu|)$ onto $\mathfrak{T l}_{s}$. Then from (3.1) we have that $\{E(s):-\infty<s<\infty\}$ is a resolution of the identity in $L_{2}(G,|\mu|)$. Further, it is easy to check that $U_{\gamma} \mathfrak{N M}_{s}=\mathfrak{M N}_{s+\psi(\gamma)}$. Hence we get $U_{\gamma} E(s) U_{-\gamma}$ $=E(s+\psi(\gamma))$. Let $V_{t}=\int_{-\infty}^{\infty} \exp (i t s) E(d s)$. Then by Stone's theorem $\left\{V_{t}:-\infty<t<\infty\right\}$ is a (strongly continuous) group of unitary operators. Further 


$$
\begin{aligned}
U_{\gamma} V_{t} & =U_{\gamma} \int_{-\infty}^{\infty} \exp (i t s) E(d s)=\int_{-\infty}^{\infty} \exp (i t s) U_{\gamma} E(d s) \\
& =\int_{-\infty}^{\infty} \exp (i t s) E(d s+\psi(\gamma)) U_{\gamma}=\exp (-i \psi(\gamma) t) V_{t} U_{\gamma} .
\end{aligned}
$$

Thus $V_{t}$ and $U_{\gamma}$ satisfy the relation (2.2). We now prove

Theorem 3.1 (MAIN Theorem [2]). Let $\mu$ be a $\phi$-analytic measure on $G$. Then $\mu$ is quasi-invariant under $\phi$.

Proof. Observe that if $\beta$ denotes the spectral measure of $U_{\gamma}$, then $(\beta(\Delta) f)(g)=I_{\Delta}(g) f(g), g \in G$, where $I_{\Delta}(g)=1$ or 0 according as $g \in \Delta$ or $g \notin \Delta$. Therefore $\beta(\Delta)=0$ if and only if $|\mu|(\Delta)=0$. Now $U_{\gamma} V_{t}$ $=\exp (-i \psi(\gamma) t) V_{t} U_{\gamma}=\chi_{\gamma}(\phi(t)) V_{t} U_{\gamma}$. Hence by Theorem 2.1, $V_{t} \beta(\Delta) V_{-t}=\beta(\Delta+\phi(t))$. Thus $\beta(\Delta)=0$ implies $\beta(\Delta+\phi(t))=0$ for all $t$ and therefore $\mu$ is quasi-invariant under $\phi$. q.e.d.

If $\Gamma=R_{d}$ (the real line with the discrete topology), it is well known $[12$, p. 30] that $G$ is the Bohr compactification $B$ of $R$ and there is continuous isomorphism $\phi$ of $R$ onto a (dense) subgroup $\phi(R)$ of $B$. The following corollary is now obvious.

COROLlary 3.1. If $\mu$ is an analytic measure on $B$, then $\mu$ is quasiinvariant under $R$.

In fact, Theorem 3.1 is not far more general than Corollary 3.1 in the sense that it could be obtained essentially using Corollary 3.1. The important part is played by the Archimedian order of $R$. One may observe that if $\phi$ is a nontrivial continuous homomorphism then the kernel of $\phi$ is either 0 or a discrete subgroup of $R$ isomorphic to the group of integers. The latter case can be essentially proved by using a variation of Theorem 2.1. In the next section, we shall therefore restrict our attention to Bohr group.

4. Quasi-invariant measures and invariant measures. Two positive regular $\sigma$-finite measures on $B$ will be called equivalent if they are mutually absolutely continuous. For any measure $\mu$ on $B$ we shall let $\mu_{t}$ denote the measure given by $\mu_{t}(A)=\mu(A+\phi(t)), A$ being a Borel subset of $B$. Let $\mu$ be a positive finite regular measure on $B$ quasi-invariant under $\phi$. It is easy to check that the Radon-Nikodym derivative $g(t, \cdot)=\left(d \mu_{t} / d \mu\right)(\cdot)$ satisfies the functional equation for a cocycle (cf. [3]), i.e., $g(t+s, x)=g(t, x) g(s, x+t)$, a.e. [ $\mu]$. Here the set of measure zero, where the equation does not hold, may vary with the pair $(t, s)$. 
THEOREM 4.1. There exists $a \sigma$-finite $\phi$-invariant measure $\nu$ equivalent to $\mu$ if and only if the cocycle $g$ is a coboundary, i.e., there exists a measurable function $h$ such that

$$
g(t, x)=\frac{h(x+\phi(t))}{h(x)}, \text { a.e. }[\mu] .
$$

Proof. Suppose there exists a $\sigma$-finite $\phi$-invariant measure $\nu$ equivalent to $\mu$. Consider

$$
g(t, \cdot)=\frac{d \mu_{t}}{d \mu}=\frac{d \mu_{t}}{d \nu}(\cdot) \frac{d \nu}{d \mu}(\cdot)=\frac{h(\cdot+\phi(t))}{h(\cdot)}
$$

where $h(\cdot)=(d \mu / d \nu)(\cdot)$. Hence $g$ is a coboundary. Conversely suppose that $g$ is a coboundary. Let $\nu(A)=\int_{A}(1 / h(x)) \mu(d x)$. Since the set $\{x: 0<h(x)<\infty\}$ is also the support of $\mu$ it is easy to check that $\nu$ is a $\sigma$-finite measure equivalent to $\mu$. To see that $\nu$ is $\phi$-invariant, we note that

$$
\begin{aligned}
\nu(A+\phi(t)) & =\int_{A+\phi(t)} \frac{1}{h(x)} \mu(d x)=\int_{A} \frac{1}{h(x+\phi(t))} \mu_{t}(d x) \\
& =\int_{A} \frac{1}{h(x+\phi(t))} \frac{d \mu_{t}}{d \mu} \mu(d x) \\
& =\int_{A} \frac{1}{h(x+\phi(t))} \frac{h(x+\phi(t))}{h(x)} \mu(d x)=\nu(A) \text {. q.e.d. }
\end{aligned}
$$

A $\phi$-invariant measure $\nu$ on $B$ need not be the Haar measure on $B$. For example, consider measure $\nu$ that is Lebesgue measure on $\phi(R)$. In view of this the following result, though elementary, is interesting.

THEOREM 4.2. If a finite nonzero measure $\mu$ is invariant under $\phi(R)$ then $\mu$ is the Haar measure on $B$.

Proof.

$$
\begin{aligned}
\hat{\mu}(t) & =\int_{B} \chi_{t}(b) \mu(d b)=\int_{B} \chi_{t}(b+s) \mu(d b), \quad s \in \phi(R), \\
& =\int_{B} \exp (i t s) \chi_{t}(b) \mu(d b)=\exp (i t s) \hat{\mu}(t) .
\end{aligned}
$$

Hence $\hat{\mu}(t)=0$ for $t \neq 0$ and $\hat{\mu}(0)=\mu(B) \neq 0$. Hence $\mu$ is the Haar measure on $B$ (or a constant multiple of it). q.e.d. 


\section{REFERENCES}

1. S. Bochner, Boundary values of analytic functions in several variables and of almost periodic functions, Ann. of Math. 45 (1944), 708-722.

2. K. de Leeuw, and I. Glicksberg, Quasi-invariance and analyticity of measures on compact groups, Acta Math. 109 (1963), 179-205.

3. H. Helson, Compact groups with ordered duals, Proc. London Math. Soc. XIV A (1965), 144-156.

4. - Invariant subspaces, Academic Press, New York, 1964.

5. H. Helson and D. Lowdenslager, Prediction theory and Fourier series in several variables. I, Acta Math. 99 (1958), 165-201.

6. - Prediction theory and Fourier series in several variables. II, Acta Math. 106 (1961), 175-212.

7. G. Kallianpur and V. Mandrekar, Representation and multiplicity of purely non-deterministic stochastic processes, Theory of Probability and its Applications $\mathbf{X}$ (1966).

8. G. W. Mackey, A theorem of Stone and von Neumann, Duke Math. J. 16 (1949), 313-326.

9. M. G. Nadkarni, On a class of measures on Bohr group, Pacific J. Math. (to appear).

10. J. von Neumann, Die Eindeutigkeit der Schrödingerschen Operatoren, Math. Ann. (1931), 570-578.

11. F. Riesz and B. Sz-Nagy, Functional analysis, Ungar, New York, 1955.

12. W. Rudin, Fourier analysis on groups, Interscience, New York, 1962.

UNIVERSITY OF MINNESOTA

\section{A NONLINEAR BOUNDARY VALUE PROBLEM}

BY R. WILHELMSEN

Communicated by H. A. Antosiewicz, May 22, 1967

1. Introduction. The main result of this paper establishes the existence of solutions of certain nonlinear two point boundary value problems for a class of nonlinear second order differential equations.

A corollary to the main theorem includes a boundary value problem recently considered by Herbert B. Keller [1] and Klaus Schmitt [2].

2. Definitions. In the following definitions let $\mathbf{S}$ stand for a point set in the $Y Z$-plane.

$$
\begin{aligned}
& A=\{S: S \text { is an arc }\}, \\
& H_{1}=\left\{S:\left(Y_{1}, Z_{1}\right),\left(Y_{2}, Z_{2}\right) \in S \Rightarrow\left(Y_{1}-Y_{2}\right)\left(Z_{1}-Z_{2}\right) \geqq 0\right\}, \\
& H_{2}=\left\{S:\left(Y_{1}, Z_{1}\right),\left(Y_{2}, Z_{2}\right) \in S \Rightarrow\left(Y_{1}-Y_{2}\right)\left(Z_{1}-Z_{2}\right) \leqq 0\right\}, \\
& J_{1}=\{S: \forall \exists(Y, Z) \in S \ni Z=N\}, \\
& J_{2}=\{S: \forall \exists(Y, Z) \in S \ni Y-Z=N\}, \\
& R=\left\{(X, Y, Z): X_{1} \leqq X \leqq X_{2},|Y|+|Z|<\infty\right\}, \\
& B_{0}=\{f(X, Y, Z): f \text { is continuous in } R\},
\end{aligned}
$$

